\title{
Cómo predecir a más personas en más ocasiones: evaluando la personalidad de las situaciones(*)
}

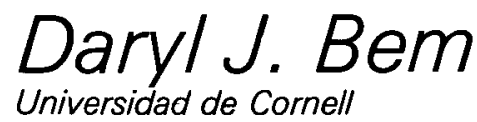

Universidad de Cornell

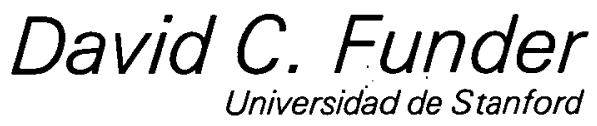

Traducción: M. ${ }^{a}$ Luisa Sánchez Bernardos.

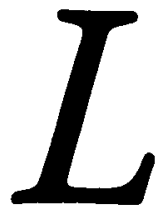

La creciente aceptación de que gran parte de la varianza de interés psicológico de la conducte podría encontrarse en la interacción entre la persona y la situación, sugiere la necesidad de un lenguaje común para la descripción tanto de las personas como de las situaciones. De acuerdo con esto, proponemos que una situación sea caracterizada por una serie de pares de plantillas de conducta, esto es, un conjunto de descripciones de personalidad (del tipo Q) de hipotéticas personas (idealesi), cada una de las cuales se asocia con una conducta concreta. La descripción del tipo $Q$ de un determinado individuo se empareja pues, con cada plantilla, y se predice que mostrará la conducta asociada con la caracterización situacional-tipo a que más se parezca. La utilidad heuristica y predictiva de esta técnica de emparejamiento, se demuestra en tres grupos de experimentos clásicos: (a) la situación de demora de la gratificación, (b) el juego de negociación estratégica, y (c) el experimento de condescendencia forada. Se muestra que esta técnica también puede utilizarse para evaluar la validez ecológica de los experimentos de laboratorio, y para probar teorias que conciemen a los fenómenos psicológicos.

Como todos sabemos, la conducta es función tanto de la persona como de la situación. Pero, a pesar de la reciente aceptación de esta perogrullada, continuamos subestimando la influencia de la situación. Como apuntan los psicólogos uintuitivos»), mostramos este sesgo de modo tan persistente que ahora se le conoce como el (error fundamental de atribucións) (Ross, 1977); y, como psicólogos profesionales, seguimos estando confundidos al mismo tiempo que alteraciones aparentemente triviales en el procedimiento experimental continúan produciendo cambios no tan triviales en las conductas de nuestros sujetos.

(*) Agradecemos a Jack Block y Walter Mischel sus observaciones sobre este articulo.

Todo el material de los datos $Q$ utilizados en esta investigación se obtuvieron de Consulting Psychologists Press, 577 College Avenue, Palo Alto, California 94306.

Con permiso de los autores y del editor. Tomado de Psychological Review, 1978, vol. 85, núm. 6, p.p. 485-501.

Copright (1978) by the American Psychological Association. Reprinted by permission. 
Por ejemplo, los niños que udemoran la gratificación») menos de un minuto cuando se colocan ante ellos durces que les gustan son capaces de esperar diez veces más si los dulces se hallan fuera de su vista (Mischel y Ebbesen, 1970, quienes, sin embargo, no están confundidos por esto). De un modo similar, los resultados de los experimentos de «condescendencia forzada», en los cuales se induce a los sujetos a comportarse de modo contraactitudinal, dependen de características de la situación sutiles y típicamente desconocidas e incontroladas (cfr. Carlsmith Collins y Helmreich, 1966; Ems y Janis, 1965; Janis y Gilmore, 1965; Rosenberg, 1965). Dada tal especificidad situacional de la conducta, no es sorprendente que la búsqueda de consistencias transituacionales en ella, se vea frustrada con tanta frecuencia (Mischel, 1968).

En la actualidad, el reciente debate sobre la existencia de tales consistencias, parece haber evolucionado hacia un acuerdo de que es la interacción entre la persona y la situación lo que explica la mayor parte de la varianza de interés psicológico de la-conducta (Ben y Allen, 1974; Endier y Magnusson, 1976; Magnusson y Endler, 1977). También es evidente que, mientras que la tarea de evaluar a las personas ha avanzado rápidamente en Psicología a lo largo de las décadas, la evaluación de las situaciones ha comenzado a recibir una atención similar sólo en fechas recientes. (Ver revisiones de Frederiksen, 1972; Moos, 1973; y Pervin, 1978). Los psicólogos ambientalistas han comenzado a investigar cómo influyen en la conducta las condiciones físicas de la situación, tales como temperatura, dimensiones espaciales y diseño arquitectónico (e. g., Kasmar, 1970). Se han analizado las organizaciones en términos de su tamaño, distribución del personal y niveles de sueldos medios; y los ambientes universitarios se han caracterizdo en términos de sus normas sociales, status socioeconómico de los estudiantes, actitudes de la Facultad, etc. Otras investigaciones han estudiado más las dimensiones psicológicas de las situaciones, tales como el grado en que son (arduas) 0 (fáciles) para los sujetos (Arseniam y Arseniam, 1948), el grado en que proporcionan vías claras $y$ directas hacia metas importantes (Fuller, 1950), o el grado en que tienden a ser "seguras», "desorganizadas», etc. (Chein, 1954). La «atmósfera» psicológica general de las instituciones ha sido evaluada obteniendo las percepciones de aquellos que habitan en estos ambientes (Moos, 1973), y se ha sugerido que las situaciones también se pueden caracterizar en términos de las conductas que tienden a elicitar en el individuo medio (Frederiksen, 1972. Block
(1971) utiliza una técnica $\mathbf{Q}$ (uambiental) para caracterizar el entorno pasado de un individuo. Y, en uno de los escasos intentos de estudiar la interacción persona-situación, Craick (1976) informa de relaciones entre variables de personalidad y entorno físico.

Sin embargo, ninguno de los métodos actuales de evaluación emplea un lenguaje descriptivo que pueda ser utilizado para caracterizar tanto a las personas como a las situaciones de un modo directamente equivalente (Murray, 1938). Creemos que el desarrollo de tal sistema descriptivo común, seria un paso importante hacia una teoria coherente de la interacción persona-situación, y es el propósito de este artículo introducir tal sistema.

El enfoque que vamos a describir -la técnica del emparejamiento de plantillas- es, de hecho, simplemente una formalización de un procedimiento que utilizamos en la vida diaria. Consideremos, por ejemplo, cómo podríamos responder a la pregunta: «¿Debería David ir a Stanford?». Observemos que esta es una cuestión típica acerca de la interacción persona-situación, no acerca de efectos principales. A David no le interesa saber cómo le iría en otras universidades, ni como es la vida en general de los estudiantes de Stanford. David necesita saber cómo sus propias características enlazan o interactúan con las características de Stanford. Sin saber nada de David, ¿cómo podríamos orientarle? Una manera corriente es describir Stanford en términos de cómo funcionan en ese ambiente varios tipos de personas (ideales): «los estudiantes que trabajan mucho, pero que son algo tímidos, tienden a obtener buenas notas e interactúan pobremente en la vida de facultad; los estudiantes brillantes y asertivos a menudo intervienen en los proyectos de investigación de la facultad, pero, en consecuencia, llevan una escasa vida social y obtienen notas por debajo de sus posibilidades; los estudiantes que....), etc. Ahora, todo lo que David debe hacer para predecir sus resultados en Stanford, es emparejar sus propias características con el conjunto de descripciones-tipo que le hemos proporcionado. Más que describir Stanford en términos de número de alumnos por clase, prestigio de la facultad, requisitos para la titulación, etc., lo hemos caracterizado en términos de un conjunto de descripciones de resultados o de pares de patrones de conducta. $Y$, llegado el momento de elegir Universidad, la predicción de David sobre sus resultados se vería grandemente facilitada por el hecho de que hemos descrito el ambiente de Standford, precisamente en el mismo sistema de lenguaje que utilizariamos para caracterizar al mismo David. 


\section{Estudios}

Lo que estamos proponiendo aquil es, pues, que las situaciones sean caracterizadas como conjuntos de pares de patrones de conducta, siendo cada patrón una descripción de un utipos ideal de persona, de la que se espera que se comporte en una dirección dada. La probabilidad de que una persona concreta se comporte de un modo determinado en una situación dada, se postula como una función monotónica creciente del empareja miento o similaridad entre las caracteristicas de dicha persona y el patron asociado con la conducta correspondiente. Como veremos, los pares de patrones de conducta pueden construirse post hoc a partir de los datos, sobre la base de las evaluaciones de los observadores, 0 se pueden derivar de una teoría (p. ej., las personas con baja tolerancia a la inconsistencia, cambiarán sus actitudes resultando, asi, un comportamiento contra-actitudinal).

Una teoría completa de la interacción persona-situación tendría, aún, que especificar cómo estos patrones se relacionan con propiedades de las situaciones evaludadas independientemente (p. ej., ¿cuáles son las propiedades funcionales de la situación de condescendencia forzada que hacen que se produrca un cambio de actitud en tales sujetos?). A menudo, este paso conceptual de las plantillas a las propiedades funcionales de la situación, puede ser bastante directo si el lenguaje descriptivo se ha elegido bien. Observemos, por ejemplo, cuán fácilmente David podría inferir muchas características del ambiente de Stanford -número de alumnos por clase, distancia que hay a la facultad, tipo de investigación que se lleva a cabo- directamente a partir de la descripción de patrones de conducta que le hemos proporcionado. $Y$, en la investigación que expondremos aquil, veremos cómo en varios contextos experimentales las descripciones de patrones de conducta dirigen rápidamente la atención a las propiedades funcionales relevantes de la situación. No obstante, sigue presente ef problema conceptual básico; $y$ volveremos sobre él al final del artículo.

\section{EL LENGUAJE DE LA EVALUACION .}

Una técnica de evaluación que sirva para caracterizar tanto a las personas como a las situaciones, necesita cumplir un cierto número de requisitos. En primer lugar debe ser lo suficientemente general como para abarcar una amplia variedad de situaciones; el lenguaje en sí mismo no debe ser específico de una situación. En se gundo lugar, debería proporcionar medidas ujpsativas» más que normativas. Como han argumentado Bem. y Alen (1974), no todos los rasgos o dimensiones de personalidad pueden aplicarse a cualquier persona e, inclur so, las diferencias individuales estriban en qué rasgos de personalidad son importantes, centrales o sobresalientes, para el individuo. Asi pues, lo que se necesita es un instrumento que determine la importancia relativa y la configuración de las variables intraindividuales más que la permanencia relativa de los individuos a través de las variables. $Y$, finalmente, el instrumento debe prestarse a si mismo a una estrategia analítica que permita comparar dos personas, dos patrones conducta-situación o un patrón y una persona globalmente en términos de su grado de «emparejamiento» o similaridad.

Un instrumento que satisface estos criterios es la Técnica 0 , que utiliza los conjuntos de items ideados por Block (1961): La Técnica Q de Clasificación de Califomia y la Técnica Q de Clasificación de California para Niños (Nota 1). Ambas constan de 100 afirmaciones descriptivas de la personalidad ( $p$. ej., ues incapaz de demorar la gratificacióny), que están clasificadas en nueve categorías ordenadas de la menos a la más característica de la persona en cuestión. Las afirmaciones que no son ni características ni no caracteristicas del individuo se colocan en categorías intermedias. Así, cada item, recibe una puntuación de 1 a 9 y se emplea una distribución simétrica con una media de 5 y una desviación típica aproximadamente de 2. Los items fueron evaluados y ampliados 'por un gran número de psicólogos clinicos y psiquiatras, y se sometieron a escrutinio tanto estadístico como empírico a lo largo de varios años; han sido revisados y reformulados varias veces. $\theta$ resultado es un conjunto de items de personalidad que cubren ampliamente el campo de la misma, emparejados con una redundancia relativa mente poco importante; más aún, existe actualmente un gran cuerpo de investigación que utiliza estas técnicas. Aunque no derivados de ninguna orientación teórica concreta, muchos de los items tienen un aire ligeramente psicodinámico y se utilizan tanto a niveles de descripción fenotípicos como genotípicos. En Block (1961) puede encontrarse una extensa descripción de la metodología de la clasificación $\mathbf{O}$ de California en particular. La Técnica $\mathbf{Q}$ de California se diseñó en principio para uso de psiquiatras y psicólogos clínicos profesionales. En consécuencia, fue necesario modificar el conjunto para que los no profesionales pudiesen clasificar sus conocimientos del mismo modo que los profesionales. Dado que se habia llevado a cabo un gran estuerzo en la re- 
dacción precisa de los items originales, nosotros los mantuvimos intactos y simplemente añadimos paráfrasis entre paréntesis debajo del item original. Por ejemplo, el item (tendencias represivas o disociativas"), ahora también incluye la paráfrasis «tiende a negar pensamientos, conflictos o sentimientos desagradables; prefiere creer que no existen»s. La Técnica 0 de California para Niños, se publicó originariamente para su utilización por no profesionales y por ello no fue necesario modificario.

Los datos de tipo $Q$ pueden ser analizados de muchos modos. E análisis más frecuente consiste en comparar dos clasificaciones $\mathbf{Q}$ por medio de una correlación de Pearson producto-momento inter-items, expresando así directa y cuantitativamente el grado de semejanza entre ambas. Se puede utilizar este procedimiento para establecer el grado de acuerdo interjueces entre dos clasificaciones que describan al mismo individuo; 0 se puede fijar el grado de similaridad entre muchos pares de upersonalidades), sometiendo las matrices de correlación resultantes a un análisis factorial inverso 0 a un análisis de clusters para obtener tipologias de personas.

Sin embargo, el procedimiento analítico más relacionado con el propósito que nos ocupa, requiere la elaboración de clasificaciones $\mathbf{Q}$ de hipotéticas personalidades ideales. Tradicionalmente se ha conseguido haciendo una lista de las concepciones de varios (expertos») de la técnica Q, de algún tipo hipotético de personalidad ideal (p. ej., el varón paranoide o la personalidad óptimamente ajustada), y asi reunir todos los juicios en un único dato $\mathbf{Q}$ (Block, 1961). Los datos $\mathbf{Q}$ de individuos reales están, pues, correlacionados con este criterio de acuerdo, para determinar su similitud con el tipo ideal. Es la lógica de este último procedimiento la que nos ocupa en el presente estudio.

En concreto, proponemos que las plantillas de la situación se definan por medio de datos 0 de cada tipo ideal de persona que se comporte de un modo conceptualmente distinto en la situación concreta de que se trate. Así, Stantord sería caracterizado por varios datos Q, cada uno de los cuales describe un tipo hipotético de es. tudiante que se comporta de un modo específico en el ambiente de Stanford. Habría un dato $\mathbf{Q}$ del estudiante que obtiene buenas notas pero que interactúa pobremente en la vida de la facultad, (utrabajadon), utimidon); otro caracterizaría al aventajado estudiante entusiasta de la investigación, (chrillante), (kasertivo),... etc. Algunos patrones de conducta podrian tener una alta generalidad transituacional; por ejemplo, las caracteristicas del estudiante con atto logro académico podrían muy bien ser casi consistentes en distintas facultades y universidades. Otros podrían ser relativamente específicos para el caso de Stanford (p. ej., las características del estudiante entusiasta de la investigación que sólo consigue notas medias, pero que es considerado por la Facultad incluso como candidato al Premio Nobel). La upersonalidad» única de la situación deriva, pues, del conjunto total de combinaciones de los pares de patrones de conducta, del mismo modo que la unicidad de un individuo deriva de su peculiar combinación de rasgos sobresalientes, algunos de los cuales están ampliamente repartidos en la pobla. ción, mientras que otros son relativamente infrecuentes.

Una vez que una situación especifica ha sido caracterizada por un conjunto de descripciones de conducta, el comportamiento de un individuo determinado en esa situación se predice correlacionando el dato $\mathbf{Q}$ de su propia personalidad con cada caracterización situacional. La correlación más alta fi. e., el emparejamiento más adecuado entre la persona y la caracterización de la situación) identifica la conducta predicha.

El modelo predice que, para cada individuo, la ordenación de las correlaciones entre la persona y el patrón situacional, seguirá el orden de la probabilidad que haya de que se realicen las conductas asociadas con los correspondientes patrones situacionales.

En este artículo aplicaremos la técnica de emparejamiento de caracterizaciones de personalidad y situación a tres grupos de experimentos clásicos. En el primer caso - la situación de demora de la gratificación- nuestro propósito principal es demostrar cómo la información de los datos $Q$, en y a partir de sí misma, puede proporcionar un valioso conocimiento de una situación experimental; cómo puede sensibilizar a los investigadores hacia características de las situaciones experimentales que son teóricamente extrañas, pero que, sin embargo, están afectando a la conducta de los sujetos; y cómo puede ayudar a señalar las fuentes de inconsistencia del comportamiento en situaciones aparentemente similares. Este estudio ilustra también cómo se pueden construir los patrones de los datos $\mathbf{Q}$ sobre bases totalmente upost hoc).

E segundo caso, el juego de negociación estratégica (p. ej., el Juego del Dilema del Prisionero) fue examinado por Bem y Lord en un estudio (Nota 2). Su trabajo, que será resumido brevemente demuestra cómo la técnica del emparejamiento de patrones puede determinar la validez ecológica de cualquier procedimiento de laboratorio que pretenda ser una situación análoga a la vida real. Su estudio ilustra también cómo se pueden construir los 
patrones sobre la base de las descripciones que los observadores hacen de la situación.

En el último caso -los experimentos de condescendencia forzada- demostramos cómo se puede emplear la técnica del emparejamiento de patrones para probar teorías opuestas sobre una situación; al mismo tiempo el estudio ilustra como los patrones-tipo se pueden deducir de una teoria formal.

\section{EVALUACION OE LAS CARACTERISTICAS DE UNA SITUACION DE DEMORA EN LA GRATIFICACION (1)}

La capacidad de un individuo para demorar la gratifica ción y ejercer formas de autocontrol adaptativo es muy importante, tanto por razones teóricas como prácticas. En consecuencia existe una amplia literatura de investigación sobre estos temas, y una diversidad de métodos para determinar las conductas relevantes (ver Block y Martin, 1955; Mischel, 1974). Por ejemplo, un primer trabajo de Mischel, uno de los principales investigadores en este área, evaluó la disposición de un individuo a renunciar a una pequeña recompensa inmediata con el fin de obtener una recompensa mayor después de un tiempo determinado. Un procedimiento, estrechamente relacionado con éste, mide la duración del tiempo que un niño es capaz de esperar para conseguir su dulce preferido, en lugar de tomar inmediatamente el dulce que le gusta menos. Aún existe otro procedimiento que mide el espacio de tiempo que un niño es capaz de esperar antes de abrir un regalo envuelto (Block, 1977).

Como el mismo Mischel ha observado (1968, p. 82) estos comportamientos tienden a ser bastante específicos de la situación: la puntuación de autocontrol conseguida por los niños en una situación, raramente correlaciona por encima de .30 con las puntuaciones obtenidas en otra situación, coeficiente tope de la consistencia que se encuentra normalmente en la investigación en Personalidad. $Y$, como generalmente sucede en este campo, rara vez está claro qué caracteristicas propias de las distintas situaciones son responsables de las inconsistencias observadas. Como veremos, es precisamente este tipo de información la que los datos 0 pueden proporcionarnos (2).

\section{METODO}

16 niiños y 13 niñas de clase media alta, con una media de edad de 4 años y 6 meses, tueron observados en una situación experimental, en la que se evaluó el tiempo que eran capaces de esperar para recibir su dulce preferido en lugar de uno que les gustaba menos, pero que estaba disponible inmediatamente. Cada niño estaba sentado enfrene de dos dulces distintos, y se le preguntaba cuál de los dos preferia. Se le djjo entonces, que el experimentador iba a estar en la habitación de al lado y que cuando volviera, el niño podría tomar su dulce preferido. Pero, si el niño prefería llamarlo, al experimentador volvería enseguida; esto podría hacerse en cualquier momento tocando el timbre colocado en la mesa; $y$ en este caso, se daría al niño el dulce que menos le gusta ba. Cada 3 minutos se recordaba al niño esta contingencia durante el tiempo de demora, y se le preguntaba si quería que el experimentador volviera entonces, 0 si preferla esperar más. Si no le llamaba para que volviera, el experimentador regresaba después de una demora de 15 minutos. 1 procedimiento fue adoptado con considerable -y.consiguiente- modificación de Mischel y Ebbesen (1970).

Los datos $\mathbf{Q}$ de los niños fueron aportados por sus padres, utilizando la Técnica $\mathbf{Q}$ de Clasificación de California para Niños (Block y Block, Nota 1). Se obtuvieron dos clasificaciones (una de cada padre) de 20 niños, y una sola clasificación de 9 de ellos. Cuando se obtuvieron dos clsificaciones por niño, se combinaron para el análisis en una puentuación media.

\section{RESULTADOS Y DISCUSION}

\section{Correlaciones de los datos 0 con la demora}

Como primer paso en el análisis, se halló la correlación entre cada uno de los 100 items del conjunto de datos 0 de los niños y sus tiempos de demora. Como puede verse en la Tabla 1, varios items correlacionaron significativamente con la demora. Además los items correlacionados positivamente ofrecen una imagen del niño que mantiene una demora larga, que, de acuerdo con Mischel, es bastante consistente con la literatura evolutiva sobre el control del yo y el comportamiento prosocial (comunicación personal; ver también, Mischel, 1974). De este modo, observamos a un niño que, por el mecanismo de la identificación (utiende a imitar y adoptar gestos caracteristicos y conductas de aquéllos a quienes admiras), adopta criterios de ejecución elevados, se vuelve protector con los demás, servicial, cooperativo, empático, considerado, solicito y capaz de desarrollar relaciones personales. Se puede encontrar evidencia 
adicional para la interpretación del control del yo en algunos de los items correlacionados negativamente, incluyendo, desde luego, el item crucial ues incapaz de demorar la gratificacióny. Por ejemplo, se ve que el niño que mantiene una demora larga es emocional. mente poco expresivo y no es inquieto o impaciente, atributos consistentes con la inhibición expresiva y motora implicadas en el desarrollo del control del yo (Mischel, 1966).

Sin embargo, el resto de la imagen expresada en la Tabla 1, introduce una nota más disonante: un retrato del niño que mantiene una demora larga como no muy inteligente, con poca fluidez verbal, no ávido de apren- der, ni abierto a nuevas experiencias -más aún, un niño que no es autoasertivo, ni alegre, interesante 0 creativo-. La relación altamente negativa entre la demora y el nivel de inteligencia es particularmente inconsistente con las teorías del control del yo, y Mischel informa que en su propio trabajo, encontró que el tiempo de demora correlacionaba positivamente con el nivel de inteligencia (comunicación personal). Verdaderamente la (gestaltb) que brota de este conjunto completo de items $\mathbf{Q}$, sugiere que el niño que es capaz de esperar en esta situación, puede ser caracterizado correctamente tanto si se dice que es aburrido, pasivo y obediente a la autoridad del adulto, como si se dice que posee una gran cantidad de autocontrol.

Tabla 1

Correlaciones entre los Items Q y las Puntuaciones de la Demora.

\section{Correlacionados Positivamente}

Tiene criterios de ejecución elevados para sí mismo $\ldots \ldots \ldots \ldots \ldots \ldots \ldots \ldots$

Tiende a imitar y adoptar gestos y conductas de aquellos a quienes admira . . . . . . .

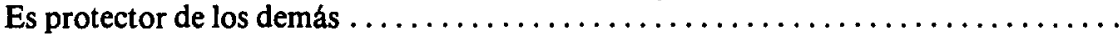

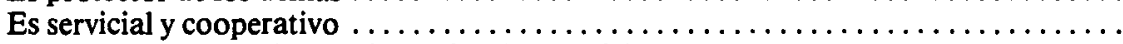

Reconoce los sentimientos de los demás (empático) $\ldots \ldots \ldots \ldots \ldots \ldots \ldots \ldots \ldots \ldots \ldots$

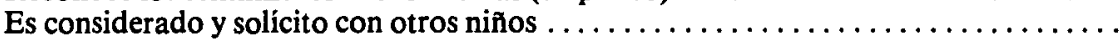

Desarrolla relaciones sinceras e íntimas

\section{Correlacionados Negativamente}

Parece tener alta capacidad intelectual

$-.62^{* * *}$
$-.56^{* * *}$
$-.50^{* * *}$
$-.49^{* * *}$
$-.47^{* *}$
$-.43^{* *}$
$-.43^{* *}$
$-.40^{* *}$
$-.37^{* *}$
$-.34^{*}$
$-.31^{*}$
$-.31^{*}$
$-.31^{*}$

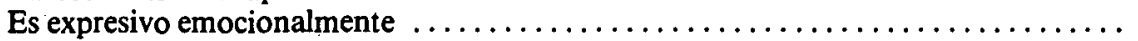

Tiene fluidez verbal, es capaz de expresar bien las ideas en palabras..............

Es curioso y explorador, ávido de aprender, abierto a nuevas experiencias . . . . . . . .

Es autoasertivo . . . . . . . . . . . . . . . . . . . . . . . . . . . . . . . .

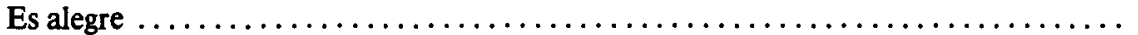

Es un niño interesante, que llama la atención $\ldots \ldots \ldots \ldots \ldots \ldots \ldots \ldots \ldots \ldots$

Es creativo en la percepción, en el pensamiento, en el trabajo o en el juego .........

Intenta que las culpas recaigan sobre los otros . . . . . . . . . . . . . . . . . . . .

Se comporta de un modo dominante con los demás $\ldots \ldots \ldots \ldots \ldots \ldots \ldots \ldots \ldots$

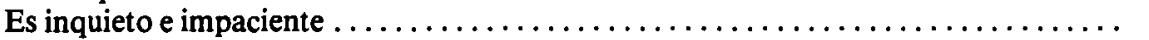

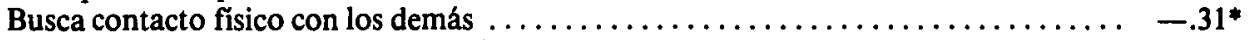

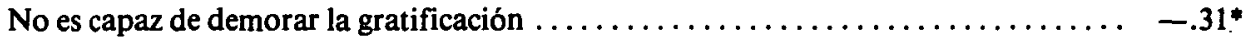


Lo que queremos hacer notar aquí es el hecho de que el cuadro de items $\mathbf{Q}$ apunta bastante directamente a las propiedades de la situación que parecen ser relevantes para los niños, y que los controlan funcionalmente. Asi, podría parecer que la presencia del experimentador y la deseabilidad social implicita en la demora, son tan rele vantes para los niños en esta situación como los diferentes atractivos de los dos items-alimento, estímulo que, en el campo teórico, se supone que es la varible controladora. En su propio trabajo, Mischel siempre hace que el experimentador salga de la habitación y, frecuentemente, introduce una condición de máxima condescendencia, como línea de control, para desestimar estos factores (Mischel, 1974; Mischel, Ebbesen y Zeiss, 1972). Como señalamos antes, el dato $Q$ puede funcionar camo un valioso instrumento para detectar caracteristicas, teónicamente extrañas, de una situación experimental que están afectando a las conductas de los sujetos.

Resulta también instructivo comparar esta imagen de los datos Q con la que resulta de un experimento de Block (1977) sobre udemora del regalo), experimento que podría parecer prácticamente idéntico al nuestro. En el experimento de Block, se mostró al niño un regalo vistosamente envuelto, y se le dijo que ibạ a permanecer cerrado hasta que él completara un puzde. La medida de la demora de la gratificación fue la duración del tiempo que el niño esperó antes de extender el brazo y coger el regalo. La Tabla 2 muestra los items $\mathbf{Q}$ que más altamente correlacionaron con la demora en este caso (3).

Como puede verse en la Tabla 2, el item ues incapaz de demorar la gratificación)y es el mejor y más sencillo pronóstico del tiempo de demora de los niños. Además, algunos items parecen apoyar la interpretación de que esta situación está, de hecho, descubriendo otra dimensión del control de impulsos: uhace proyectos para el futurou); (ues atento y capaz de concentrarse); , ues reflexivo, piensa y delibera antes de actuarn).

Tabla 2

Correlaciones entre los Items Q y los Tiempos de Demora del Regajo

(De Block, 1977)

Item

Correlaciones positivamente

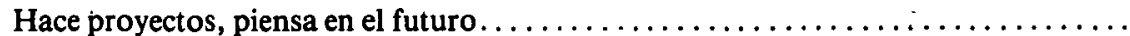

Es reflexivo, piensa y delibera antes de actuar. . . . . . . . . . . . . . . . . . .

Se implica mucho en todo lo que hace.$\ldots \ldots \ldots \ldots \ldots \ldots \ldots \ldots \ldots \ldots \ldots \ldots \ldots$

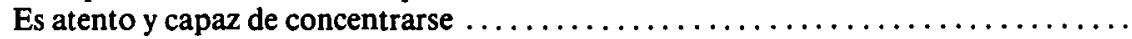

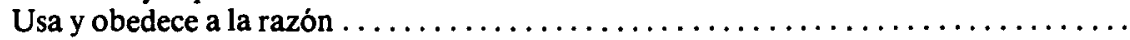

Es tímido y reservado; le cuesta establecer contactos sociales ................

Es protector con los demás . . . . . . . . . . . . . . . . . . . . . . . . . . .

Tiende a guardarse para si sus pensamientos y sentimientos $\ldots \ldots \ldots \ldots \ldots \ldots \ldots$

Tiene una vida interior rica.

\section{Correlacionados negativamente}

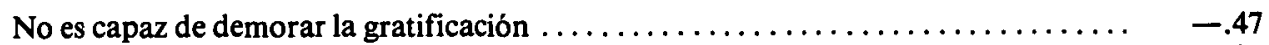

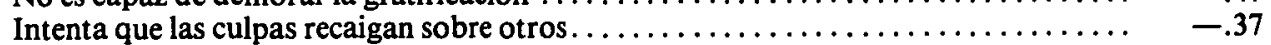

Tiene rápidos cambios de humor; lábil emocionalmente $\ldots \ldots \ldots \ldots \ldots \ldots \ldots \ldots \ldots \ldots \ldots \ldots \ldots$

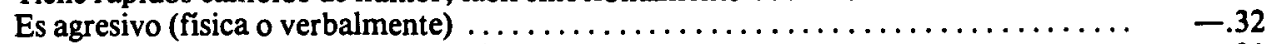

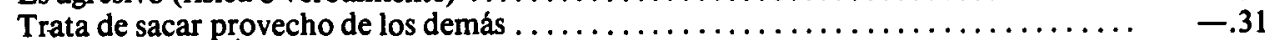

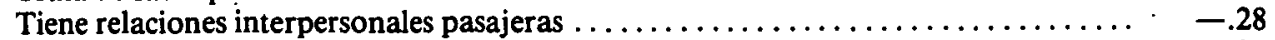

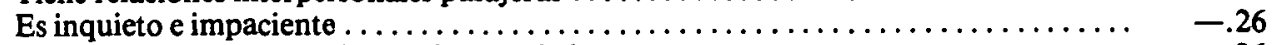

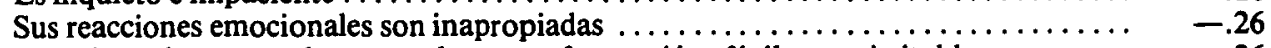

Reacciona desmesuradamente a la menor frustración; fácilmente irritable ........ $\quad-.26$

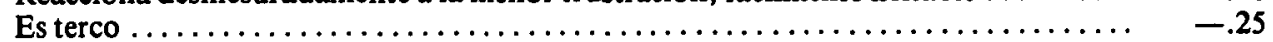

Expresa sentimientos negativos directa y abiertamente $\ldots \ldots \ldots \ldots \ldots \ldots \ldots \ldots \ldots \ldots \ldots$

NOTA: Todas las correlaciones en esta tabla son cálculos basados en las correlaciones separadas por sexos referidas por Block (1977). Nosotros hemos reproducido aquí los 20 ítems con la correlación media más alta. 
Sin embargo, está claro que las Tablas 1 y 2 nos proporcionan retratos muy distintos del niño de larga demora; incluso los items relevantes para el constructo del control del yo muestran escaso solapamiento en las dos tablas; y el grupo de items aburrido-pasivo-obediente que surge en nuestro experimento, está completamente ausente en los datos de Block. Lo que tenemos aquil, pues, son dos situaciones que parecen conceptualmente equivalentes, pero que funcionalmente son bastane distintas, y podría parecer que son diferentes los subgrupos de niños que están demorando ef refuerzo en los dos casos. Normalmente, de lo único que nos damos cuenta es de que en dos situaciones teóricamente similares la conducta es decepcionante- mente inconsistente. Sin embargo, reuniendo los datos del tipo $Q$, se puede ver con esmerado detalle la naturaleza de la inconsistencia y sacar inferencias plausibles acerca de su origen a partir de las carac. teristicas que se solapan en las dos situaciones.

Cuando menos, la información de tipo 0 proporciona una guia valiosa para el experimentador, que necesita volver a diseñar un procedimiento experimental, de modo tal que le sirva para sus propósitos. Incluso los psicólogos que no tienen particular interés en las diferencias individuales per se, deberían dar la bienvenida a esta entrada en la fenomenología de las situaciones que ellos han creado.

\section{ELABORACION DE LOS PATRONES-TIPO}

Como vimos antes, en nuestro procedimiento, un patrón-tipo es él mismo un dato $Q$, una ordenación 〈ideal» de los items $\mathbf{Q}$ utilizados para caracterizar a los individuos cuya conducta vamos a predecir. La construcción de un patrón implica asignar valores numéricos a cada uno de los items en el conjunto $Q$, y hay varias posibilidades consistentes con nuestro modelo general de llevar esto a cabo. Por razones sustantivas y estéticas hemos preferido elaborar los patrones ajustando todas las puntuaciones de acuerdo con la siguiente fórmula:

$$
\mathrm{Q}=\mathrm{M}_{\mathbf{i}}+\mathrm{R}_{\mathrm{i}} \sigma_{\mathbf{i}}
$$

donde $Q_{1}$, es el valor ajustado, et valor del patrón del item (ij), $M$ y $\sigma_{i}$ son respectivamente la media y la desviación tipica del item (ui) de la muestra total de sujetos, y $p_{i}$ es el peso del factor que refleja la urelevancia del item (iin para la conducta criterio con que vamos a predecir.

Supongamos, por ejemplo, que $\mathrm{p}_{\text {j }}$ toma el valor del coeficiente de correlación entre el item (ij) y la conducta de demora de los niños en nuestro estudio. Si el item no correlaciona con la conducta de demora $\left(r_{i}-p_{i}-0\right)$, el valor ajustado del item en nuestro patrón tipo es simplemente igual a la media de este item en la muestra de sujetos. Pero ahora consideremos el item (tiene criterios de ejecución elevados para sí mismo»), que correlaciona +.48 con la coducta de demora. Este item se ajustará .48 de una desviación típica por encima de la media de grupo, y el dato 0 resultante será más parecido a los datos $\mathbf{Q}$ del que demora mucho, que a los datos $\mathrm{Q}$ del que demora poco. Los items que correlacionan negativamente con la conducta criterio se ajustarán, desde luego, por debajo. Como implica la fórmula, cuanto mayor sea la varianza de un item en la muestra, más radicalmente tendrá que ser ajustado.

Puesto que la relevancia de un item está totalmente contenida en las $p_{\text {, }}$, es posible separarlo de la información normativa sobre una muestra concreta. Por. ello, la información relevante puede ser transportada de un sujeto a otro, utilizando la información normativa (las Ms y las $\sigma_{i} s$ ) de la misma muestra. (Por ejemplo, utilizando el mismo conjunto de $p_{i} s$ construimos habitualmente patrones diferentes para los dos sexos). Esto nos permite generar patrones-tipo que mantengan sus caracteristicas propias de la situación, al mismo tiempo que caracterizar con más detalle la muestra concreta de individuos cuya conducta vamos a predecir.

Deberá observarse que la relevancia de los items está contenida en las magnitudes relativas de las p,s, y por tanto, sus magnitudes absolutamente arbitrarias. Si se pretende una teoría audaz, que realce o desprecie marcadamente los items relevantes a partir de las medias de la muestra, las ps deberán ordenarse para cubrir un amplio rango; si, por el contrario, pretendemos una teoría tímida, en la cual el patrón no se aleje mucho de la composición de los datos $\mathbf{Q}$ del sujeto, las ps deberán ordenarse para cubrir un recorrido limitado. Por razones menos estéticas, nosotros hemos ordenado los pesos en la investigación expuesta en este artículo en un recorrido de -2 a +2 . La distribución vigente empleada con la Técnica $\mathbf{Q}$ de Clasificación de California, tiene una media de 5 y una desviación típica aproximada- 


\section{Estudios}

mente de 2 (exactamente 209 ) en una escala de 9 puntos. Asi, el item máximamente relevante $\left(p_{1}=-2\right.$ con estos parámetros podría desplazarse 2 desviaciones típicas o 4 puntos desde una categoría media (5) a la categoría umás caracteristica) (9), o a la umenos característicau) (1). Esto nos pareció bien.

Finalmente, debería observarse que, aunque la fórmula para la elaboración de los patrones permite que los pesos de los items relevantes (p) varíen continuamente, nosotros hemos utilizado en nuestra investigación un esquema de ponderación todo-0-nada. Asi, en el presente estudio, dimos a todos los items de la Tabla $\mathrm{r}$ (los items que correla cionaron significativamente con el tiempo de demora) un peso de $+20-2$, dependiendo del signo de su correlación, y al resto un valor de 0 . Esta decisión es consistente con la investigación reciente, que demuestra que la tarea más importante en la elaboración de cualquier buen modelo predictivo es identificar las variables relevantes y conseguir sus efectos en la dirección correcta: más allá de esto, todos los esquemas de ponderación son equivalentes, incluyendo los pesos elegidos aleatoriamente o los pesos unidad (p. ej.. Dawes y Corrigan, 1974; Wainer, 1976, 1978). Además, dado el pequeño tamaño de la muestra del presente estudio, sería ilusorio conceder más importancia a los valores reales de correlación mostrados en la Tabla 1.

\section{PREDICCION Y VALIDACION CRUZADA}

Una vez que se han elaborado los pares de patrones de conducta para una situación, la conducta de un individuo concreto se predice correlacionando su clasificación Q personal con cada patrón situacional, y aventurando la'predicción de que mostrará la conducta asociada con la correlación más alta. Obsérvese que las correlaciones comparadas en este proceso se han obtenido todas a partir del mismo individuo; no es ilustrativo comparar directamente una correlación de un patrón de un individuo con la obtenida por otro, porque cada correlación contiene dos componentes diferentes: la semejanza del sujeto con el individuo (medio), y la semejanza del sujeto con el perfil específico del patrón (ct. Cronbach, 1955). Puesto que los patrones son simplemente variaciones ajustadas de la media o de los da tos $\mathbf{Q}$, el sujeto más semejante al individuo medio de la muestra tendrá, en general, correlaciones mayores con todos los patrones de la situación. De esta manera, el individuo que más semejanza tiene con un patrón concreto, no es necesariamente aquél que mostrará con más probabilidad la conducta asociada.

Para deducir una puntuación de semejanza que sirva para comparar individuos, necesitamos sustraer de la correla ción del patrón de cada individuo, su correlación con la media de los datos 0 ajustados (4). Es esta puntuación de simila ridad (ipsativa) la que refleja el componente de semejanza único al perfil del patrón específico; y en consecuencia, ésta es la puntuación que se predice que estará positivamente correlacionada con el tiempo de demora de los niños en el presente estudio.

Utilizando el procedimiento anteriormente descrito, los patrones que caracterizan al niño ideal de larga demora se construyeron por separado para niños y niñas, y cada dato 0 de los niños se correlacionó con el patrón apropiado a su sexo. Se restó la correlación de cada niño con el conjunto de datos $\mathrm{Q}$ de su propio sexo, y la puntuación en el patrón de similaridad resultante se correlcionó con el tiempo de demora. Esto anojó una correlación de .75 1 < $<001$, prueba de una cola). Este valor está desde luego aumentado, por el hecho de que los patrones se han probado con los mismos datos utilizados para identificar los items relevantes, procedimiento que aumenta el efecto del azar. En conse cuencia, volvimos enseguida a la técnica de la doble validación cruzada: Dividimos la muestra en dos mitades empare jadas según el sexo y las puntuaciones de demora. Se volvieron a calcular las correlaciones de los items-conducta, y se elaboraron nuevos patrones por separado pero cada mitad de la muestra. (En la de estas plantillas, no se asignaron pesos de cero a ninguno de los 20 items de mayor correlación con el tiempo de demora; esto correspondió a una correlación con un valor absoluto de .40 o más.) Los datos 0 de cada niño se correlacionaron, después, con el patrón de su propio sexo, que había sido deducido de la submuestra que no contenia a ese niño. Se calcularon de nuevo las puntuaciones de semejanza y se correlacionaron con los tiempos de demora de los niños.

E resultado de este procedimiento fue un coeficiente de validación cruzada de $.45(p<.009$, prueba de una cola), medida no contaminada por el poder de los patrones para predecir el tiempo de demora en esta situación. La disminución de la correlación de .75 a .45 no es excepcional cuando se utilizan muestras tan pequeñas ins - 15 y 14 en las dos submuestras).

En nuestra opinión lo verdaderamente importante del presente estudio es la misma lista de items $Q$ relevantes. En el contexto del descubrimiento, es esto lo que da al experimentador el conocimiento que necesita. El procedimiento de elaboración de patrones y su validación cruzada tiene aquí, principalmente, una función relativa al ucontexto de justificaciónn), previniéndonos de la sobreinterpretación de items 0 concretos que pueden haber tenido significación estadistica por azar. En el presente caso, el procedimiento de emparejamiento de patrones no añade nada importante 
al conocimiento obtenido por un simple vistazo de las Tablas 1 2 ; $ү$, nuestro propósito aqul era principalmente ilustrativo, esto es, exponer las características generales analíticas $y$ técnicas de nuestro modelo en el contexto de un ejemplo específico. Volvemos ahora a una situación en la cual pueden ocurrir varias conductas cualitativamente diferentes, y, por tanto, se requieren varias plantillas para caracterizar la situación. Más aún, los patrones se elaboraron a priori en este estudio y no (post hoc). Bajo estas circunstancias es cuando el valor de la técnica de emparejamiento de personas y patrones situacionales resulta más notable.

\section{COMO EVALUAR LA VALIDEZ ECOLOGICA DE SITUACIONES EXPERIMENTALES}

La cuestión de la validez ecológica (ise comportan las personas en la vida real como lo hacen en nuestros laboratorios?) es central para la psicologla social experimental y la posibilidad de que la respuesta pueda ser (noo) es central para la reciente crisis de confianza en este campo (Baumrid, 1964; Ome y Holland, 1968; McGuire, 1973; Ring, 1967). Los investigadores han intentado normalmente establecer la validez externa de sus procedimientos de laboratorio de dos modos fundamentalmente. La primera estrategia, con más frecuencia propuesta que llevada a cabo, es llevar en tandem experimentos de laboratorio y estudios de campo, paralelos conceptualmente, para confirmar directamente el isomorfismo asumido. La segunda estrategia es demostrar la validez de constructo de los conceptos evaluados en el laboratorio, esto es, mostrar que el comportamiento se relaciona con otras variables en el sentido que indica la teoría. Por ejemplo la suposición de que el juego de lanzar dardos es una situación que elicita motivación de logro, se ve reforada por los resultados de que los individuos con atta motivación de logro se mantienen a una distancia mo. derada de la diana, tal y como la teoría predecía (McClelland, 1958). De forma parecida, la confianza que se pone en un paradigma de validez externa, se incrementa si variables como las diferencias individuales se relacionan con la conducta de laboratorio de una manera teóricamente convincente.

Como han sugerido Bem y Lord (Nota 2i, la técnica de emparejamiento de patrones persona-situación, proporciona una novedad, un método más directo para determinar el grado en el cual el comportamiento de los sujetos en el laboratorio coincide con su comportamiento en la vida diaria. Para probar lo expuesto, Bem y Lord investigaron el juego de la negociación estratégica, una situación experimental que ha sido frecuentemente criticada por su falta de validez ecológica (p. ej., Knox y Douglas, 1971; Nemeth, 1972; Pruitt, 1967). La versión más conocida de la negociación estratégica es el Juego del Dilema del Prisionero; recordemos que en este juego se mide simultáneamente a dos sujetos que tomen una serie de decisiones independientemente uno de otro y sin comunicación entre ellos. Si los dos escogen la res. puesta "cooperativa», su recompensa conjunta es alta; si los dos seleccionan la respuesta "competitiva" su recompensa conjunta es baja: si un jugador escoge la respuesta cooperativa y el otro la competitiva, este último (el umalou) gana puntos o dinero a expensas del cooperador.

Bem y Lord utilizan la, asi llamada, versión «descompuesta) del juego de decisiones introducido por Messick y McClintock (1968). Estos juegos permiten a los investigadores salirse de la simple dicotomía cooperativo-competitivo, $y$ distinguir tres estrategias distintas de juego: La estrategia $\mathrm{J}$, en la cual un jugador intenta maximizar las ganancias conjuntas de ambos jugadores; la estrategia $\mathbf{O}$ en la que un jugador intenta maximizar su propia ganancia absoluta; $y$ la estrategia $R$ en la que un jugador intenta maximizar su ganancia en relación con el otro jugador (5).

Bem y Lord construyeron patrones de conducta para la situación del juego, suministrando a cinco jueces una descripción escrita de las tres estrategias junto con la lista de datos $Q$. Cada juez recorría la lista y daba un valor a cada item, en un rango de valores que iba de +2 (umuy característico de la persona que sigue esta estrategia»), pasando por 0 (uno relevante, ni muy caracteristico ni poco característico de la persona que sigue esta estrategian) a -2 (kmuy poco caracteristico de la persona que sigue esta estrategia)l. Por ejemplo, al item «se comporta de una forma dada con los demásy) se le asignó un valor +1.6 por los jueces que estaban caracterizando la estrategia $\mathrm{J}$, la estrategia en la que los jugadores intenta maximizar las yanancias de ambos, esto es, las suyas propias y las del otro jugador. Se encargó explícitamente a los jueces que no manejaran teorias de Personalidad, que no especularan acerca de la remoto correlación entre personalidad y comportamiento, sino que juzgaran únicamente si un item podía caracterizar o no el comportamiento implicado en la estrategia. El valor medio dado a cada item por los cinco jueces, se convierte entonces en el peso $\left(p_{1}\right)$ de ese item en el algoritmo 


\section{Estudios}

generador del patrón anteriormente descrito. Este procedimiento se repitió para las tres estrategias, obteniendo para cada una un patrón característico de la hipotética persona ideal que la seguiría.

Bem y Lord reclutaron sujetos para participar en un experimento real, juego de negociación estratégica, y obtuvieron datos $\mathbf{Q}$ de los propios sujetos y de uno de sus compañeros de habitación. Los datos $Q$ de este úttimo se correlacionaron con uno de los tres patrones-tipo. Los resultados apoyan la validez ecológica de este juego: La correlación entre los datos $Q_{y}$ el patrón que caracteriza la estrategia real adoptada por el sujeto, era significativamente más alta que la que habia con los patrones que caracterizaban las estrategias atternativas. Verdaderamente estos individuos se comportaban en la vida real como lo hacian en el laboratorio experimental.

Antes mencionábamos que la información de los datos Q es sumamente valiosa en si misma y más allá de sí misma, y esto era también cierto en el estudio de Bem y Lord, donde se proporcionaba a los sujetos un (änsights que excedia ampliamente del éxito del procedimiento de emparejar patrones. Por ejemplo, de acuerdo con el dato de su compañera de habitación, la chica que sigue la estrategia $R$ en el laboratorio lla estrategia en la que la meta fundamental es derrotar al otro jugadorl es sutilmente negativista, tiende a socavar y obstruir o sabotear, mantiene a la gente a distancia, evita relaciones interpersonales próximas, no se comporta de una manera simpática o considerada, $y$ así sucesivamente. En marcado contraste, estas mujeres se caracterizan a sí mismas como tendentes a suscitar agrado y aceptación en la gente, con encanto personal, femeninas $y$ con elegancia y presencia social. No produce sorpresa que sus compañeras de habitación evaluaran a estas chicas significativamente más bajo de lo que ellas se evaluaban a si mismas en el item uconoce bien sus propias motivaciones y comportamientow. Se observa que los datos $Q$ han facilitado aquí tres clases distintas de información: percepciones del compañerola, autopercepciones y discrepancias entre las dos.

Voivvemos ahora a una utilización más formal de la técnica del emparejamiento de patrones: la verificación de las teorías que compiten entre sí.

\section{CONTRASTACION DE TEORIAS ALTERNATIVAS EN LA SITUACION DE CONDESCENDENCIA FORZADA (6)}

El experimento de condescendencia forzada requiere que el individuo defienda actitudes contrarias a sus pro- pias posiciones. $\theta$ resultado clásico es que subsiguientemente a su condescendencia (y bajo condiciones teóricamente especificadas), los individuos exhiben actitudes que están más cerca de las posiciones que han defendido de lo que lo estaban sus actitudes inciales. Varias teorías ofrecen explicaciones de este efecto. La Tearía de la Disonancia Cognoscitiva, fuente original del paradigma, asegura que un estado aversivo de disonancia suscitado por la discrepancia entre su conducta y sus actitudes contrarias, motiva a los sujetos a cambiar de actitudes (p. ej., Festinger y Carlsmith, 1959). La Teoría de la Auto-percepción (Bem, 1967, 1972) propone que los sujetos observan su propio comportamiento de defensa de la posición designada y entonces infieren sus actitudes finales a partir de este comportamiento, de la misma manera que to haría un observador externo. Un tercer grupo de teorías pone el énfasis en las demandas que la situación conlleva sobre la autopresentación, y sugiere que las actitudes finales que muestra el sujeto están motivadas, principalmente, por un intento de causar una determinada impresión en el experimentador (Tedeschi, Schlenker y Bonoma, 1971), apreherisión de evaluación (Rosenberg, 1965, 1969), o el deseo de resaltar una «identidad establecida» concreta tanto ante si mismo como ante los demás (Alexander y Knight, 1971).

En el presente estudio dedujimos patrones de datos 0 para cada una de estas tres perspectivas teóricas, patrones-tipo de una persona hipotética que, de acuerdo con cada teoría, mostraría el mayor cambio de actitud en la situación de condescendencia forzada. Las predicciones basadas en el patrón se contrastaron con los resultados de un experimento real, permitiendo que las teorlas compitieran entre sí en la predicción de la varianza de las diferencias individuales.

\section{METODO}

17 varones y 15 mujeres, todos ellos estudiantes, participaron en un experimento clásico de condescendencia forzada, en el cual se les inducia a escribir sobre un tema contra-actitudinal, bajo condiciones diseñadas para incrementar el grado en el que sentian que tenian fibertad de elección para aceptar o rechazar nuestra petición. Al finalizar, se compararon las actitudes sobre el tema discutido en sus escritos; con las actitudes que habian expresado varias semanas antes de empezar el experimento. El experimento fue una réplica exacta de la condición de «elección» del estudio de condescendencia forzada comentado en Bem y McConnell (1970). Unas cuan- 
tas semanas después, se pagó a cada sujeto 2 dólares para que trajera a dos conocidos suyos que estuvieran dispuestos a proporcionar datos confidenciales sobre él, utilizando la versión modificada de la Técnica $\mathbf{Q}$ de California descrita anteriormente (Block, 1961). Estos evaluadores, a los que también se pagó 2 dólares, eran amigos y/o compañeros de habitación de los sujetos, y se conocían aproximadarrante desde hacia un año y medio. Los datos $\mathbf{Q}$ obtenidos para cada sujeto se promediaron juntos para obtener un dato conjunto (7).

\section{CONSTRUCCION DE PATRONES TIPO: TEORIA DE LA DISONANCIA COGNOSCITIVA}

Después de una cuidadosa lectura de varias de las exposiciones de la Teoría de la Disonancia Cognoscitiva, formulamos los siguientes tres tipos de personas que mostrarían un mayor cambio de actitud de acuerdo con la teoria:

TIPO A.-Personas que con más probabilidad están alerta a inconsistencias entre sus creencias, actitudes y comportamientos.

TIPO B.-Personas que con cierta probabilidad encuentran aversivas las inconsistencias cognitivas y están motivadas a acabar con ellas.

TIPO C.-Personas cuyas creencias y actitudes no están probablemente ancladas e imbricadas en una estructura integrada de creencias. (Por ello reducirían probablemente la disonancia por medio del cambio de actitudes, mejor que por medio de otros métodos).

Se dio esta clasificación y la lista completa de datos 0 a tres psicólogos que habian sido activos investigadoresiteóricos en el paradigma de condescendencia forzada: un teórico de la disonancia (J. Merrill Carlsmith), un teórico de la autopercepción (Daril J. Bem), y un psicólo. go que ha publicado ampliamente sobre ambas teorias (Mark R. Lepper). Cada uno de estos tres jueces revisaron la lista Q, preguntándose a si mismos si cada item era pertinente a alguno de los tres tipos $\gamma$, si asi era, dando a ese item un signo positivo o negativo seguido de la letra que designa qué tipo de persona caracteriza to caracteriza negativamednte si se le asigna un negativol. Por ejemplo, los tres jueces dieron al item uestá incómodo con las incertidumbres y complejidades») un valor $+B$, que indica que es positivamente caracteristico de ipersonas que con cierta probabilidad encuentran aversivas las inconsistencias cognitivas y están motiva das a acabar con ellas». El item ues introspectivo y se preocupa de sí mismon, fue uno de los seleccionados como caracteristico de la persona que con más probabilidad está alerta a inconsistencias cognitivas (Tipo A); $y$ et item «tiende a ser indeciso en varios aspectos») fue se leccionado como característico de la persona cuyas creencias tienen a no estar imbricadas en una estructura integrada de creencias (Tipo C).

Cualquier item seleccionado como relevante por unno de los tres jueces, se utilizó en la elaboración del patrón tipo, a menos que uno de los otros jueces le hubiera da do un signo opuesto, en cuyo caso se omitía. Bajo este criterio se seleccionaron $\mathbf{3 0}$ de los 117 items, como relevantes para el patrón de la teoría de la disonancia. A estos items se les dio un peso de $+2 y-2$, y se introdujeron en el algoritmo generador del patrón tipo descrito antes en este articulo. Los patrones eran distintos para hombres $y$ mujeres.

\section{CONSTRUCCIONES DE PATRONESTIPO: TEORIA DE LA AUTOPERCEPCION}

En los informes publicados de la teoría de la auto-percepción (Bem, 1965, 1967, 1972), formulamos los cuatro tipos siguientes de personas que mostrarian, probablemente, cambio de actitudes:

TIPO A.-Personas que creen que, en general, su conducta refleja sus verdaderas creencias y actitudes les decir, que su conducta generalmente (dice la verdads).

Tipo B. -Personas que en general no son conscientes o no están sensibilizadas a sus propios estados, incluyendo sus creencias y actitudes iniciales ly necesitarian, por tanto, contar con más indicios externos para inferir sus creencias y actitudes).

Tipo C.-Personas cuyas creencias y actitudes no están, probablemente ligadas a otra evidencia o no están imbricadas en una estructura integrada de creencias (y, por tanto, basarian sus creencias y actitudes finales en la conducta auto-observada exclusivamente).

Tipo D.-Personas que, en general, se dejan influir por un comunicante persuasivo (puesto que se supone que la auto-persuasión es funcionalmente similar a la persuasión interpersonal, Bem, 1964).

Los mismos tres jueces evaluaron la relevancia de los items $\mathbf{Q}$ de estos tipos, obteniendo un total de 39 items relevantes. Por ejemplo, los jueces seleccionaron el item (da conducta es normalmente expresión de sus propios sentimientos, actitudes o creencias), como caracteristico de la persona Tipo A. El item uconoce bien sus propias motivaciones $y$ conductan, fue juzgado negativa 
mente característico del Tipo B, y «es crítico, escéptico, no fácilmente impresionables), se consideró negativa mente característico del Tipo D. Se elaboraron de nuevo patrones para ambos sexos de acuerdo con el algoritmo.

\section{CONSTRUCCION DE PATRONES-TIPO: TEORIA DE LA AUTO-PRESENTACION}

Como vimos antes, la teoría de la auto-presentación es, en efecto, nuestra denominación para una amalgama de varias posiciones que participan de la idea de que el cambio de actitudes observado en un experimento de condescendencia forzada, refleja un intento de los sujetos por proyectar imágenes deseables al experimentador, a ellos mismos 0 a ambos. Las siguientes citas explican brevemente la situación de condescendencia forzada propuesta por tres de estas teorías:

«En el laboratorio, los sujetos saben que están participando en un experimento, $y$ que el experimento ha sido autorizado por la universiad, el departamento y los científicos de la facultad... Además, la caricatura del científico es que está :... impresionado ante todo por la racionalidad, conșistencia y coherencia. Con pistas tan formidables para la presentación apropiada de uno mismo, no es de extrañar que los resultados obtenidos sean consistentes en el tiempo (Tedeschi et al., 1971, p. 691).D

«Incluso cuando el sujeto esté convencido de que su ajuste personal no está siendo directamente estudiado, probablemente pensará que el experimentador está, no obstante, acechando para detectar cualquier conducta que indique un ajuste pobre o falta de madurez. (Rosenbert, 1965, pp. 28-29)..)

«Se predice la conducta si se puede alcanzar la identidad más favorabje a partir de una de las afternativas de conducta que son posibles bajo ciertas circunstancias. La gente espera convertirse en la clase de persona mejor evaluada. (Alexander y Knight, 1971, p. 79/).

Desgraciadamente la deducción de las predicciones de las diferencias individuales a partir de estas teorias no es muy clara. Naturalmente, el patrón que se construye para la teoria general, apresaria al hipotético individuo ideal que está más alerta a las normas sociales, y que con más probabilidad se ajusta a ellas; pero la dirección de la conducta de esta persona - cambio o estabilidad de actitudes- está totalmente indeterminada. Excepto para la posición de Tedeschi et al.(1971), que sostiene que los sujetos estarán motivados a mostrar consistencia, y por lo tanto cambio de actitudes, las otras dos teorías dejan abierta la cuestión de qué imagen exacta tra tará de ofrecer un sujeto dado. Para algunos individuos, por ejemplo, la identidad más favorable en esta situación, sería la de una persona servicial y cooperativa, esto es, el «buen») sujeto. Si estos individuos son susceptibles a las normas de la situación, mostrarán cambio de actitudes. Por otro lado, algunos individuos pueden creer que parecerán más ajustados o maduros si mantienen sus opiniones originales y se resisten a la persuasión inherente a esta situación. Desde luego, el varón muy ligado al rol sexual masculino, que es susceptible a las normas culturales de la masculinidad, se resistirá probablemente al cambio de actitudes para parecer asertivo, inconformista e, incluso, rebelde. De manera parecida, la mujer muy ligada al rol sexual femenino elegirá la sumisión y el cambio de actitudes como la conducta que ofrece la imagen más valorada. (De hecho hay razones para suponer que las normas de los roles sexuales deben estar implicadas en este tipo de situación, porque las correlaciones entre el cambio de actitudes y varios items del tipo $\mathrm{Q}$ están fuertemente moduladas por el se$x_{0}$. En consecuencia, elaboramos patrones modulados por el sexo para seleccionar primeramente los items que caracterizan a los dos tipos de personas siguientes:

Tipo A.-Personas que son serviciales, cooperativas $y$, que en un experimento, se esforzarían por ser (buenos) sujetos.

Tipo B.-Personas que están pendientes y probablemente conformes con las normas sociales, incluyendo las relativas al rol sexual.

Por ejemplo, la persona más servicial, cooperativa (Tipo A) se caracterizaba positivamente por items tales como use comporta de una manera simpática y considerada), y negativamente por items tales como uligeramente negativista, tiende a socavar y obstruir o saboteans. La persona que está pendiente y que fácilmente coincide con las normas sociales, incluyendo las normas de los roles sexuales (Tipo B), se caracterizaba por items tales como «es socialmente receptiva a un amplio abanico de signos interpersonales), "(se comporta con un estilo $y$ manera masculinolfẹmenino», y uparece ser consciente de la impresión que provoca en los demási. Se seleccionó un total de 22 items eñ una lista de 117 (8).

Después construimos los patrones asignando pesos de $-2 y+2$ a los items seleccionados, y dando un valor de cero a todos los demás, Para el patrón femenino se dijo 
un peso de +2 a todos los items que ean positivamente caracteristicos del Tipo $A$ o del tipo $B$; a los negativa. mente característicos se les dio un peso de -2 Asi, se predice que la mujer hipotética que es servicial y cooperativa ylo que está conforme fácilmente con las normas sociales, mostrará un cambio de actitudes. Para el patrón masculino, se dio un peso de +2 a todos los items positivamente ciracterísticos del Tipo $A$, o negativamente característicos del Tipo $B$; a los items negativamente característicos del Tipo A y positivamente característicos del Tipo $B$ se les dio un valor de -2 En otras palabras, se predice que el hombre hipotético que es servicial y cooperativo, como su contrapartida femenina, mostrará un cambio de actitudes; pero el hombre que está pendiente y se conforma fácilmente a las normas sociales, incluyendo las del rol sexual, se predice que no mostrará cambio de actitudes. Es importante observar que no estamos prediciendo que las mujeres mostrarán más cambio de actitudes que los hombres; de hecho, no es asi. Predecimos, sin embargo, que los individuos que están muy ligados a su rol sexual se comportarán en esta situación según el estereotipo sexual. Esta es nuestra suposición uad hoc», que la conducta estereotipada feme nina supone aquil cambio de actitudes, y la masculina, estabilidad; una suposición no contenida en las teorías mismas. El lector tendrá que juzgár cuán plausible es nuestra teorla hibrida, asl como su fidelidad a las tres subteorías en las que se incluye.

\section{TABLA 3}

Correlaciones entre las Puntuaciones del patrón de Similaridad y el Cambio de Actitudes para los Patrones derivados de la Teoría de la Disonancia Cognoscitiva, la Teoría de la Auto-Percepción y la Teoría de la Auto-Presentación.

Teoría de la Disonancia Cognoscitiva

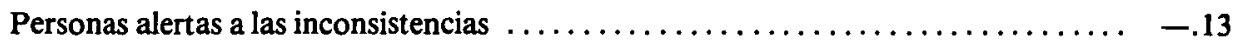

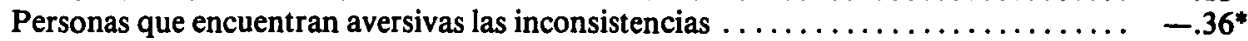

Personas cuyas creencias no están integradas en una estructura $\ldots \ldots \ldots \ldots \ldots \ldots \ldots . .20$

Patrón-tipo completo $\ldots \ldots \ldots \ldots \ldots \ldots \ldots \ldots \ldots \ldots \ldots \ldots \ldots . .25$

\section{Teoría de la Auto-Percepción}

Personas que creen que su conducta refleja sus actitudes $\ldots \ldots \ldots \ldots \ldots \ldots \ldots \ldots, \quad .01$

Personas insensibles a sus propios estados $\ldots \ldots \ldots \ldots \ldots \ldots \ldots \ldots \ldots \ldots \ldots \ldots . .24$

Personas cuyas creencias no están integradas en una estructura $\ldots \ldots \ldots \ldots \ldots \ldots \ldots . \quad 20$

Personas generalmente influenciables por la persuasión $\ldots \ldots \ldots \ldots \ldots \ldots \ldots \ldots, \quad .27$

Patrón-tipo completo $\ldots \ldots \ldots \ldots \ldots \ldots \ldots \ldots \ldots \ldots \ldots \ldots \ldots . .32 *$

\section{Teoría de la Auto-Presentación}

Personas que son serviciales, cooperativas, «buenos» sujetos . ...............

Personas fácilmente eonformes con las normas, incluyendo las del rol sexual .......

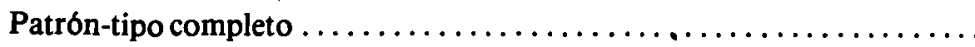

NOTA: Para la teoría de la disonancia cognoscitiva versus la teoría de la auto-percepción, $t(29)=2.64, p<.02$; para la teoría de la disonancia cognoscitiva versus la teoría de autopresentación, $t(29)=3.19, \mathrm{p}<.005$; para la teoría de la auto-percepción versus la teoría de la auto-presentación, $t(29)=1.20$, ns.

$* \mathrm{p}<.10$ (dos colas)

$* * \mathrm{p}<.02$ (dos colas)

*** p <.005 (dos colas). 


\section{Estudios}

\section{RESULTADOS Y DISCUSION}

Nuestro experimento de condescendencia forzada obtuvo el efecto clásico: Hubo un cambio significativo de actitudes hacia la posición defendida en los escritos por la muestra completa, $t(31), p<.01$ (prueba de dos colas). Hubo también grandes diferencias individuales. Por ejemplo, 19 sujetos mostraron cambio de actitudes en la dirección esperada, pero los 13 restantes no lo hicieron.

Con el objeto de probar la capacidad de cada una de las teorias para predecir estas differencias individuales, el dato $\mathbf{Q}$ de cada sujeto se correlacionó con el patron-tipo de su propio sexo construido para cada teoría. Como en el estudio de demora de la gratificación, se modificó la correlación, se hizo uipsatival, sustrayendo de esta la correlación entre los datos $\mathbf{Q}$ del sujeto y la media de to. dos los sujetos del mismo sexo, obteniendo una puntuación de similaridad que permitta la comparación entre dos sujetos. Cuanto más alta sea esta puntuación, ma yor será la similaridad del sujeto con el sujeto típico mo. dificador de actitudes descrito por esta teoría. A continuación estas puntuaciones se correlacionaron en todos los sujetos con las puntuaciones de cambio de actitudes. Cuanto más alta sea esta correlación, mejor predicción del cambio de actitudes hará la teoría. Los resultados se muestran en la Tabla 3.

Como podemos ver en la Tabla 3, la teoría de la diso. nancia cognoscitiva no tuvo éxito al predecir el cambio de actitudes; las puntuaciones del patrón de similaridad correlacionaron negativamente con las del cambio $(r=-25)$. La teoria de la auto-percepción tuvo un éxito moderado $(r-.32, p<.10$, dos colas); y la teoría de la auto-presentación fue la que más éxito tuvo $(r-.53$, $\mathrm{p}<.005$, dos colas). Como se ve al pie de la tabla, tanto la teoría de la auto-presentación como la de la auto-percepción hacen una predicción significativamente mejor que la de la disonancia cognoscitiva (prueba de las diferencias entre las correlaciones de una misma muestra, McNeman, 1969, p. 158), pero no difieren significativamente entre sí.

También es posible observar en la Tabla 3 qué componentes concretos de las tres teorias contribuyen a su éxito (o fracaso) en la predicción del cambio de actitudes. Por ejemplo, el segundo componente de la predicción de la disonancia, que afirma que, slas personas que encuentran aversivas las inconsistencias y están motivadas a acabar con ellass) deberían mostrar un cambio de actitudes mayor, parece venirse abajo -las predicciones generadas por este componente correlacionaron bastan- te negativamente con el cambio de actitudes ( $r=-.36)$-. Los dos mejores componentes de la predicción de la autopercepción son los aspectos de la teorla de la autopersuasión $(r-27)$, $y$ la idea de que las personas relativamente insensibles a sus propios estados deberían mostrar el mayor cambio de actitudes $(r-24)$. Pero los resultados de una de las predicciones más directas de la teoría de la auto-percepción -que las personas que creen que su conducta refleja sus propias creencias deberían mostrar más cambio de actitud- es, en el mejor de los casos, decepcionante. Finalmente puede observarse que los dos componentes de la teoría de la auto-presentación contribuyen significativamente a su éxito predictivo, aportando un incremento en credibilidad superior a las pruebas de $t$ totales que figuran al pie de la Tabla 3.

Es importante, sin embargo, tener claro lo que el presente estudio demuestra y to que no demuestra. Demuestra que el patrón de la auto-presentación explica una porción mayor de la varianza individual en el cambio de actitudes de los demás patrones. No demuestra, empero, que la teoría de la autopresentación sea la única teoría correcta para esta situación. Desde luego, si verdaderamente creemos en las interacciones entre persona y situación, tendremos que estar preparados para aceptar que las tres teorías podrían ser correctas pero para diferentes personas. De este modo, los resultados del presente estudio sólo pueden implicar que la mayoría de las personas que cambiaron de actitud en nuestra muestra, estaban, de hecho, motivadas por factores de auto-presentación. Sin embargo, una minoría de los que cambiaron podrían ser uauto-perceptores》 0 ureductores de disonanciam. Incluso la correlación negativa obtenida por la teoria de la disonancia cognoscitiva, no excluye la posibilidad de que hubiera reductores de disonancia en nuestra muestra, sino que podían quedar enmascarados por los modificadores de actitud de un tipo diferente que mostraban una tolerancia, aunque irrelevante, de la disonancia.

Existe otra razón estrechamente relacionada con la anterior para no sacar conclusiones excesivas de esta demostración ilustrativa: algunas teorías son más capaces que otras de generar predicciones sobre las diferencias individuales, y una mayor capacidad para predecir efectos individuales, no implica una mayor capacidad para predecir los efectos de la situación. Así, nosotros trabajamos únicamente con la condición de elección del experimento tipico de condescendencia forada, y nuestra competición requeria que las teorías dieran cuenta de la 
varianza intracelda (efectos de la persona); probablemente, los que han ganado y los que han perdido correrian una suerte distinta en una batalla sobre la varianza intercelda (efectos de la situación). Después de todo, la ma yoría de las teorías psicosociológicas consiguen su sta tus prediciendo diferencias entre tratamientos, incluyendo las teorías probadas en nuestro estudio; lo que ocurre es que nosotros las hemos forzado para que jueguen el desacostumbrado papel de teorias de Perso. nalidad.

Esto nos lleva a una refiexión amplia: Si las teorias de Personalidad han encallado porque se limitan a los efectos de la persona en un mundo poblado de interacciones persona-situación, no deberiamos ser más benevolentes con las teorias que se limitan a los efectos de la situación. Tal vez la existencia de la técnica del empa rejamiento de patrones alentará a estas teorías a florecer como verdaderas teorias de la interacción persona situación. No podemos pensar en un instrumento más refinado para abonar este crecimiento.

\section{COMO PREOECIR A MAS PERSONAS EN MAS OCASIONES}

Al final de su articulo: «Cómo predecir a Algunas Personas en Algunas Ocasiones: La Búsqueda de Consistencias Transituacionales en la Conductan, Bem y Allem (1974) afirman los siguiente:

«La creencia de que la interacción persona-situa ción explicará la mayor parte de la varianza de interés psicológico de la conducta, (ha) movido a varios escritores recientes a enfatizar el hecho de que la evaluación de la personalidad debe comenzar a prestar seria atención a las situaciones. Estamos de acuerdo. Pero, nosotros hemos preferido hacer hincapié en la idea, perfectamente simétrica, pero quizá más sutil, de que la evaluación de la personalidad también debe comenzar a prestar seria atención a las personasy.

Debe quedar claro que el programa de investigación comentado en este artículo representa nuestro intento de (prestar seria atención a las situaciones». No es fortuito que hayamos descubierto que tenemos muchas cosas que decir a los psicólogos sociales experimentales, incluyendo a aquéllos que no tienen ningún interés en las variables de personalidad, y muy poco que decir a los personólogos -aparte de eso tan aburrido de que, sí, las situaciones influyen en la conducta-.
La siguiente fase de nuestra investigación, concebida e iniciada por nuestro colega de Stanford, Curt Hoffman, está diseñada para responder al problema iperfectamente simétrico, pero quizá más sutilly de prestar seria atención a las personas. Para Bem y Allen uprestar seria atención a las personasy) significa un acercamiento al enfoque de la personalidad centrado en la persona (ldiográ fico), más que al centrado en las variables (Nomotético). De este modo, seleccionamos la técnica 0 precisamente porque nos permite estudiar la relevancia y la configura ción de las variables intraindividuales, más que la posición relativa de los individuos a lo largo de las variables (Block, 1961). En consecuencia, el propósito de Hoffman es el que ahora perseguimos: la imagen en espejo del proyecto aquí expuesto. Más que caracterizar situaciones con patrones formulados en el lenguaje de la personalidad, deberíamos ahora caracterizar a las personas con patrones formulados en el lenguaje de las situaciones. Más que describir una situación en términos de cómo se comportarla un grupo de hipotéticas personas ideales en ella, deberíamos describir a una persona en términos de cómo se comportarla en un conjunto de situaciones ideales hipotéticas. Por ejemplo, dariamos a un sujeto un conjunto de items que describieran propiedades de la situación (p. ej., (nno está estructurada), udemanda 0 exige independencia), $y$ (sse caracteriza por la presencia de figura(s) de autoridad)/, con la instrucción de. construir una clasificación $\mathbf{Q}_{0}$ un patrón que describiera la situación ideal que provoca la máxima ansiedad; un segundo patrón podría caracterizar la situación hipotética que le produciria la máxima confianza en sí mismo; y asi sucesivamente. Se podría entonces predecir el comportamiento del individuo en una situación original, emparejando estas características de la situación con los diferentes patrones de la persona, haciendo la predicción de que el sujeto mostrará la conducta asociada con el patrón a que más se asemeje. Esta perspectiva responde directamente al requerimiento de Bem y Allen: avanzar y ser idiográfico.

Este proyecto se dirige también al problema conceptual suscitado anteriormente en este artículo: ¿cómo pueden relacionarse nuestros patrones-tipo con las propiedades de estas situaciones evaluadas independientemente? ¿Cómo, por ejemplo, pueden identificarse de modo sistemático aquellas propiedades funcionales de la situación de condescendencia forzada que hacen que ésta provoque cambio de actitudes en ciertos tipos de individuos? El uso combinado de la técnica $\mathbf{Q}$ de personalidad que hemos empleado aquí y la nueva técnica $\mathrm{Q}$ si- 


\section{Estudios}

tuacional que ahora proponemos, podria permitimos, en teorla, cerrar este vacio conceptual de nuestro modelo.

La teoria definitiva de la interacción persona-situación, nos permitiría, pues, barajar ambas técnicas 0 y prede- cir después cómo esta persona hipotética se comportaria en esta hipotética situación. Nuestra próxima monografía: Cómo predecir a Todas las Personas en Todas las Ocasiones, está, sin embargo, aún en preparación.

\section{Notas de referencia}

1. BLOCK J., y BLOCK, J. H. Instructions for the California Chid Q-Set (Memorandum). Berkeley: University of California, Institute for Personality Assesment and Research, 1969.

2. BEM, D. J. y LORD, C. G. Template-Matching proposal for probing the ecological validity of experimental settings in social psychology. Manuscrito sin publicar, Stanford University, 1978.

\section{Referencias}

ALEXANDER, C. N., \& KNIGHT, G. W. Situated identit and social psychological experimentation. Sociometry, 1971, 34, 65-82.

ARSENIAN, J., \& ARSENIAN, J. M. Though and easy cultures: A conceptual analysis. Psychiatry, 1948, 11, 377-385.

BAUMRID, D. Some thoughts on ethics of research. After reading Milgram's «Behavioral study of obedience". American Psychologist, 1964, 19, 421-423.

BEM, D. J. An experimental analysis of beliefs and attitudes. (Tesis doctoral, Universidad de Michigan, 1964). Dissertation Abstracts, 1964, 25, 3723. (University Microfilms N. ${ }^{\circ} 64-12.588$ ).

BEM, D. J. An experimental analysis of self-persuasion. Journal of Experimental Social Psychology, $1965,1,199-218$.

BEM, D. J. Self-perception: An alternative interpretation of cognitive dissonance phenomena. Psychological Review, 1967, 74, 183-200.

BEM, D. J. Self-perception theory. En L. Berkowitz (Ed.), Advances in experimental social psychology (Vol. 6). Nueva York: Academic Press, 1972.

BEM, D. J. \& ALLEN, A. On predicting some of the people some of the time: The search for cross-situational Consistences in behavior. Psychological Review, 1979, 81, 506-520.

BEM. D. J., \& McCONNELL, H. K. Testing the self-perception explanation of dissonance phenomena on the salience of premanipulation attitudes. Journal of Personality and Social Psychology. 1970, 14, 23-31.

BLOCK J. The Q-sort method in personality assessment and psychiatric research. Springfield. Ill.: Charles C. Thomas, 1961.

BLOCK, J. Lives through time. Berkeley, Calif.: Bancroft Books, 1971.

BLOCK, J. Advancing the psychology of personality Paradigmatic shift or improving the quality of re: rearch? En D. Magnusson \& N. S. Endler (Eds.). Personality at the crossroads: Current issues in interactional psychology. Hillsdale, N. J.: Erlbaum, 1977.

BLOCK, J., 8 PETERSEN, P. Some personality correlates of confidence, caution and speed in a decision situation. Journal of Abnormal and Social Psychology, 1955, 51, 34-41.

BLOCK, J. H., \& MARTIN, B. Predicting the behavior of children under frustration. Journal of Abnormal Social Psychology, 1955, 51, 281-285.

CARLSMITH, J. M., COLLINS, B.E., \& HELMREICH, R. L. Studies in forced compliance: I. The effect of presure for compliance on attitude change produced by face-to-face role playing and anonymous essay writing. Journal of Personality and Social Psychology, 1966, 4, 1-13.

CHEIN, I. The environment as a determinant of behavior. Journal of Social Psychology, 1954, 39, 115-127.

CRAIK, $\mathbf{X}$. H. The personality research paradigm in environmental psychology. En S. Wagner, S. B. Cohen \& B. Kaplan (Eds.). Experiencing the environment. Nueva York: Plenum, 1976. 
CRONBACH, L. J. Processes affecting scores on «under standing of others» and «assurned similitary». Psychological Bulletin, 1955, 52, 177-193.

DAWES, R. M., \& CORRIGAN, B. Linear models in decision making. Psychological Bulletin, 1974, 81, 95-106

ELMS, A., \& JANIS, I. L. Counter-norm attitudes induced by consonant versus dissonant conditions of roleplaying. Journal of Experimental Research in Personality, 1965,1, 50-60.

ENDLER, N. S., \& MAGNUSSON, D. Interactional psychology and personality. Washington, D.C.: Hemisphere Publising, 1976.

FESTINGER, L., \& CARLSMITH, J. M. Cognitive consequences of forced compliance. Journal of Abnormal and Social Psychology, 1959, 58, 203-210.

FREDERIKSEN, N. Toward a taxonomy of situations. American Psychologist, 1972, 27, $114-123$.

FULLER, J. L. Situational analysis: A classification of organism-field interactions. Psychological Review, $1950,37,3-18$.

GOUGH, H. G. The Adjective Check List as a personality assessment research technique. Psychological Reports, $1960,0,107-122$.

GOUGH, H. G. Conceptual analysis of psychological test scores and other diagnostic variables. Journal of Abnormal Psychology, 1965, 70, 294-302.

JANIS, I. L., \& GILMORE, J. B. The influence of incentive conditions on the success of role playing in modifying attitudes. Journal of Personality and Social Psychology, 1965, 1, 17-27.

KASMAR, J. V. The development of a usable lexicon of environmental descriptors. Environment and Behavior, $1970,2,153-169$.

KNOX, R. E. \& DOUGLAS, R. L.Trivial incentives, marginal comprehension, and dubious generalizations from Prisioner's Dilemma studies. Journal of Personality and Social Psychology, 1971, 20, $160-165$.

MAGNUSSON, D., \& ENDLER, N. S. (Eds.). Personality at the crossroads: Current issues in interactional psychology. Hillsdale, N. J.: Erlbaum, 1977.

McCLELLAND, D. C. Risk taking in children with high and low need for achievement. En J. W. Atkinson (Ed.), Motives in fantasy, action and society. Princeton, N. J.: Van Nostrand, 1958.

McGUIRE, W. J. The yin and yang of progress in social psychology: Seven koan. Journal of Personality and Social Psychology, 1973, 26, 446-456.

McNEMAR, Q. Psychological statistics (4th ed.). Nueva York: Wiley, 1969.

MESSICK, D. M., \& MCCLINTOCK, C. G. Motivational basis of choice in experimental games. Journal of Experimental Social Psychology, 1968, 4, 1-25.

MISCHEL, W. Theory and research on the antecedents of self-imposed delay of reward. En B. A. Maher (Ed.), Progress in experimental personality research (Vol. 3). Nueva York: Academic Press, 1966.

MISCHEL, W. Personality and assessment. Nueva York: Wiley, 1968.

MISCHEL, W. Processes in delay of gratification. En L. Berkowitz (Ed.) Advances in experimental social psychology (Vol. 7). Nueva York: Academic Press, 1974.

MISCHEL, W., \& EBBESEN, E. B. Attention in delay of gratification. Journal of Personality and Social Psychology, 1970, 16, 329-337.

MISCHEL, W., EBBESEN, E. B. \& ZEISS, A. Cognitive and attentional mechanism in delay of gratification. Journal of Personality and Social Psychology, 1972, 21, 204-218.

MOOS, R. H. Conceptualizations of human environments. American Psychologist, 1973, 28, $652-665$.

MURRAY, H. A. Explorations in personality. Nueva York: Oxford, 1938.

NEMETH, C. A critical analysis of research utilizing the Prisoner's Dilemma paradigm for the study of bargaining. En L. Berkowitz (Ed.), Advances in experimental social psychology (Vol, 6) Nueva York: Academic Press, 1972.

ORNE, M. T., \& HOLLAND, C. H. On the ecological validity of laboratory deceptions. International Journal of Psychiatry, 1968, 6, 282-293.

PERVIN, L. A. Definitions, measurements, and classifications of stimuli, situations, and environments. Human Ecology, 1978, 6, 71-105.

PRUITT, D. G. Reward structure and cooperation: The decomposed Prisioner's Dilemma Game. Journal of Personality and Social Psychology, 1967, 7, 21-27.

RING, K. Experimental social psychology: Some sober questions about some frivolous values. Journal of Experimental Social Psychology, 1967, 3, 113-123.

ROSENBERG, M. J. When dissonance fails: On eliminating evaluation apprehension from attitude measurement. Journal of Personality and Social Psychology, 1965; 1, 18-42.

ROSENBERG, M. J. The conditions and consequences of evaluation apprehension. En R. Rosenthal \& R. W. Rosnow (Eds.), Artifacts in behavioral research. Nueva York: Academic Press, 1969.

ROSS, L. The intuitive psychologist and his shortcomings: Distortions in the attribution process. En L. Berkowitz (Ed.), Advances in experimental social psychology (Vol. 10). Nueva York: Academic Press, 1977.

TEDESCHI, J. T., SCHLENKER, B. R. \& BONOMA, T. V. Cognitive dissonance: Private ratiocination or public spectacle? American Psychologist, 1971, 26, 685-695.

WAINER, H. Estimating coefficients in linear models: It don't make no nevermind. Psychological Bulletin, $1976,83,213-217$.

WAINER, H. On the sensitivity of regression and regressors. Psychological Bulletin, 1978, 85, $267-273$. 


\section{Estudios}

\section{Notas}

(1) Por su ayuda en llevar a cabo este estudio, nos gustaría dar las gracias a Ruenell Adams, Julie Axelrod, Joni Christiansen, Colleen Kelley, Bill Knickel, Steve La Brie, Jim Pretorius, Barbara Wolcott y al Director Dee Irwin, asi como al personal del Bing Nursery School de la Universidad de Stanford.

(2) Deberá advertirse que el uso descriptivo de los datos que vamos a presentar aquí no parten de nosotros, sino que han sido empleados en programas de investigación por Block y sus colaboradores durante muchos años (p. ej., Block y Petersen, 1955; Block, 1977). Gough (1960, 1965) hace un uso parecido de la Lista de Adjetivos. En nuestra opinión esta técnica nunca ha recibido la atención que se merece, ni ha sido totalmente apreciado su valor heuristico.

(3) Los datos de Block son de niños más pequeños que los de nuestro estudio (3.5 años vs 4.5 años).

(4) Si las varianzas de los dos conjuntos de correlaciones son bastante diferentes, es conveniente normalizar cada conjunto antes de sustraer uno de otro. En nuestro estudio, las varianzas fueron casi idénticas, como lo fueron los resultados cuando se utilizaron puntuaciones normalizadas.

(5) Para designar las estrategias se ha mantenido la denominación original. Asl, la estrategia $J$ (del inglés JOINT = conjunta); la estrategia $O$ (del inglés $O W N=$ propia); y la estrategia $R$ (del inglés RELATIVE = relativa, en relación con). ( $N$. del $T$.).

(6) Agradecemos a Nancy Jamison su ayuda en el curso de este estudio; y a J. Merril Carlsmith y Mark $R$. Lepper su ayuda en la identificación de los items de los datos $Q$ para los patrones.

(7) Además de los 100 ítems estándar de la Técnica $Q$ de Clasificación de California, escribimos 17 items especificos de las teorias sometidas a prueba en este estudio. Por ejemplo, el ftem "está incómodo con las contradicciones entre sus creencias, actitudes y valores» se diseño para caracterizar a la persona que deberia mostrar un cambio de actitud, de acuerdo con la teoría de la Disonancia Cognoscitiva. Debe notarse, sin embargo, que los resultados cambian muy poco si estos ttems se omiten del análisis.

(8) Los autores sirvieron como jueces para esta plantilla. Damos las gracias a C. Normal Alexander, que nos próporcionó en este contexto una valiosa guia en el uso de su teoría de la identidad establecida. 